\title{
Assessment of Factors Contributing to TB Treatment Adherence among Patients on TB Treatment in Kano State, Nigeria: A Case Study
}

\author{
Jennifer Anyanti, Selema Akuiyibo*, Omokhudu Idogho, Stephen Ohuneni, Chinwoke Isiguzo \\ Society for Family Health, Abuja, Nigeria \\ Email: *sakuiyibo@sfhnigeria.org
}

How to cite this paper: Anyanti, J., Akuiyibo, S., Idogho, O., Ohuneni, S. and Isiguzo, C. (2020) Assessment of Factors Contributing to TB Treatment Adherence among Patients on TB Treatment in Kano State, Nigeria: A Case Study. Journal of Tuberculosis Research, 8, 209-222. https://doi.org/10.4236/jtr.2020.84019

Received: October 10, 2020

Accepted: November 13, 2020

Published: November 16, 2020

Copyright $\odot 2020$ by author(s) and Scientific Research Publishing Inc. This work is licensed under the Creative Commons Attribution International License (CC BY 4.0).

http://creativecommons.org/licenses/by/4.0/

\begin{abstract}
Background: The WHO recommended DOTS has been proven to be very effective in the treatment of tuberculosis (TB) patients. However, despite its effectiveness, non-adherence to the treatment regimen remains a major concern in TB management. This has great consequences which include persistent infectiousness, higher rates of treatment failure, continued transmission, drug resistance, and untimely death. The aim of this study is to examine the factors and behavioural patterns that contribute to treatment adherence among TB patients in Kano State, Nigeria. Methods: A qualitative study design which included two focus group discussions (FGDs) and four key informant interviews (KIIs) were adopted for this study. In total, 20 participants were interviewed comprising 16 focus group discussion participants (8 patients on treatment and 8 individuals who have been cured) divided into two groups and four key informant interviews with 4 health workers in the selected treatment facilities. Data analysis was done by translating and transcribing the responses from the FGDs and KIIs. The transcribed data was analysed using a thematic framework procedure. Results: It was observed that having a good treatment supporter who was effective and very supportive was the most important factor contributing to treatment adherence. Other identified factors that contributed to treatment adherence included: good communication by health personnel, social support from relatives and communities, little or no side effects from drug usage, low cost of transportation to the health facilities, a positive mind set towards drug usage and treatment, support and motivation from health workers, feelings of wellness during treatment, and a balanced diet. All the respondents agreed that treatment adherence had far reaching effects on positive treatment outcomes. Conclusion: For better treatment adherence, a competent treatment supporter should be available for TB patients. Health care workers should ensure that effective
\end{abstract}


communication and a cordial relationship do exist with TB patients. Also, TB patients should be properly counselled before the commencement of their treatment regimen to help them maintain a positive mind-set towards treatment. Finally, TB interventions should provide adequate social support for patients.

\section{Keywords}

Tuberculosis, DOTS, Treatment Adherence, Treatment Outcome

\section{Background of the Study}

Nigeria was listed by the World Health Organisation (WHO) as being among the 30 high burden countries for tuberculosis (TB), and ranking $7^{\text {th }}$ among the first 10. In Nigeria, the total TB incidence was estimated as 429,000 people (219 per 100,000 population) by WHO in 2018 , with about 130,000 deaths occurring from the disease not including deaths from HIV/TB coinfections [1].

Despite its high morbidity and mortality rates, tuberculosis is treatable and curable. WHO has recommended the Directly Observed Treatment Short course (DOTS) for the treatment of tuberculosis-where patients are directly observed while taking their drugs and supported throughout the treatment period. The treatment of tuberculosis for successful outcomes requires at least six months of uninterrupted usage of prescribed first line TB drugs which could be challenging for many patients. The benefits of TB treatment adherence include treatment success and reduction in the risk of transmission. On the other hand, non-adherence has negative consequences such as persistent infectiousness and higher rates of treatment failure, continued transmission, drug resistance, and death [2]. Non-adherence to treatment among TB patients increases the likelihood of developing the drug-resistant strain of the disease, which is more deadly and difficult to treat as higher drug dosages are required.

To combat and reduce the burden of tuberculosis in Nigeria, the National Tuberculosis and Leprosy Control Programme (NTBLCP) was created to reduce TB incidence and increase notification. Several multilateral and bilateral donors such as the United States Agency for International Development (USAID) and Global Fund have also contributed considerably to this cause. Being the most populous state in the country [3], Kano State is among the three states with the highest burden of tuberculosis in the country. Kano state has an estimated TB Prevalence of 45,725 cases (prevalence rate, 330/100,000) and TB case notification target of 6000 cases as of 2018 [4].

As a response to the commitment made by world leaders at 2017 United Nations General Assembly (UNGA) high-level meeting to end TB in 2030, the Kano State Government, in partnership with USAID and other agencies came up with strategies to combat this disease in the State [5]. In partnership with the State Ministry of Health and NTBLCP, the Sustaining Health Outcomes through the Private Sector (SHOPS) Plus TB Project was started in Kano to focus on ac- 
tive TB case finding and increase notification through private health facilities, including Proprietary Patent Medicine Vendors (PPMVs), Community Pharmacies (CPs), and stand-alone laboratories.

As the awareness of tuberculosis increases and more cases are identified and notified, there is the need to improve treatment adherence to increase successful treatment outcomes and reduce mortality among TB patients. To achieve optimal treatment adherence in the management of TB patients, it is expedient to understand factors contributing to treatment adherence while understanding the behavioural patterns that engender treatment adherence. This is necessary to inform programming and policy decisions on treatment adherence and serve as evidence to advise new patients on appropriate behavioural patterns and create positive attitudes towards treatment adherence among TB patients.

Previous researches conducted on factors contributing to TB treatment interruption in Nigeria found out that factors such as: distance of patients from treatment sites, lack of knowledge of the duration of treatment, low quality of support from treatment supporters and smoking habits contributed to non-adherence to treatment and increased the likelihood of treatment interruption [6] [7].

Although many studies have been conducted in Nigeria on non-adherence of TB treatment, few studies have focused on treatment adherence among TB patients in a high burden location such as Kano State. The peculiarity of the State's demographics and the culture of its people make this study necessary.

\section{Objectives of the Study}

This study aims to achieve the following: identify the factors contributing to treatment adherence, examine the behavioural patterns that aid treatment adherence and to assess the effect of adherence on treatment outcomes among TB patients in Kano State.

\section{Methodology}

\subsection{Study Area}

The study was carried out in Kano State, which is situated in North West Nigeria, with a population of about 13.4 million people; its major inhabitants are the Hausa/Fulani people [8]. It is the second most industrialized city after Lagos with a variety of businesses and industries. It is one of the three highest TB burdened States in Nigeria with Lagos and Oyo as the other two [4]. Kano has numerous public and private health facilities spread across its 44 Local Government Areas (LGAs) with most of these facilities as DOTS centres. The SHOPS Plus Project alone supports about 204 private health facilities across 26 LGAs with TB case finding and treatment [9].

\subsection{Study Population and Sampling Method}

This study was conducted among individuals who were beneficiaries of the SHOPS Plus TB Project implemented in Kano State. Participants' selection was 
purposive, the study included patients on treatment, those already cured or have completed treatment and TB healthcare workers from selected health facilities in the State. Ten (10) facilities with a patient volume of at least $25 \mathrm{~TB}$ patients receiving TB treatment monthly were included in this study and an average of 4 participants were selected per facility. In total, 45 individuals were invited for this study. However, only 20 persons ( 8 patients on treatment, 8 cured persons and $4 \mathrm{~TB}$ healthcare workers) eventually participated in the study owing to COVID-19 prevention lockdown measures which were still in place in Kano State during the data collection period. Participants were involved in a face-to-face interview to understand their treatment adherence patterns and their attitude towards treatment as well as to identify factors that contributed to their treatment adherence. TB health workers were interviewed as key informants to provide insights on the topic including about challenges faced in administering strategies to ensure treatment adherence.

\subsection{Study Design \& Data Collection Method}

This study adopted a qualitative research design, aimed at understanding the factors that contributed to treatment adherence among the TB patients. The research instruments used were Focus Group Discussion (FGD) Interview guides and Key Informant (KII) Interview guides which included sets of questions in both English and Hausa Languages aimed at understanding the research question. The questions in the interview guides sought to understand the participants' perspective towards $\mathrm{TB}$ treatment, behaviours and factors that contributed to treatment adherence and effects of TB treatment adherence on treatment outcome. Two FGDs were conducted among TB patients currently on treatment and individuals who were already cured. Each FGD lasted 45 minutes in the SFH Kano State office and comprised of eight participants per group. Similarly, four KIIs were conducted among four healthcare workers to obtain a better understanding of the subject matter. During the FGDs and KIIs, notes were taken in each session and an audio recording also is done to capture all the points raised during the sessions. The researchers paid keen attention to the discussions in each interview session to identify new information and detect data saturation points.

A Research Assistant (RA) was trained to assist with data collection and facilitation of the sessions. Prior to the actual interviews, the RA reviewed the research questions, interview guides and steps of the interview. The RA took notes during the sessions and ensured that the recordings were done appropriately; by ensuring that each recording was back-played immediately after the completion of each session. Interviewer-related bias was addressed by continuously discussing and negotiating the content of key words, broader concepts, and units of meanings with the research assistants.

\subsection{Data Analysis Technique}

The researchers discussed and clarified the content of each recording. The notes 
enabled the researchers to read about each participant's view and correctly input their quotes into the document. At the end of each day, recordings were transcribed, responses were coded and grouped into sub-themes in line with the research questions which were then analysed by two data coders and analysts. The codes were stored in a code book that was developed using Statistical Package for Social Sciences (SPSS) where the codes and themes were written. Each coded response was further grouped under three central themes and analysed to ensure adequate interpretation of the sub-topics. Few points were highlighted and quoted in the report to place emphasis and increase understanding. Transcribed data was manually analysed using thematic analysis model suggested by Braun and Clarke (2006) [10], Figure 1 which involved six main stages: familiarization, initial coding, generating themes, validity and reliability of themes, defining and naming themes, and interpretation and reporting.

\subsection{Ethical Consideration}

Ethical approval was obtained from Kano State Ministry of Health Research Ethics Committee prior to the conduct of field activities. Participants were not induced or enticed into participating in the research. Participation was voluntary and verbal informed consents were obtained from each of the participants. The participants were not exposed to any harmful or dangerous substance or activity, just strictly interviews. It was ensured that there was no form of discrimination against the participants due to their health status and all participants were adequately protected from risks of infection or re-infection by giving them protective face masks and ensuring interviews were conducted in a spacious and ventilated venue.

All participants' identities were kept confidential; only first names were used during interviews to ensure strict confidentiality and privacy. The confidentiality of information provided by the participants was ensured.

\section{Results and Discussion}

\subsection{Socio-Demographic Characteristics of Participants}

The demographic characteristics of participants of the study included age, gender,

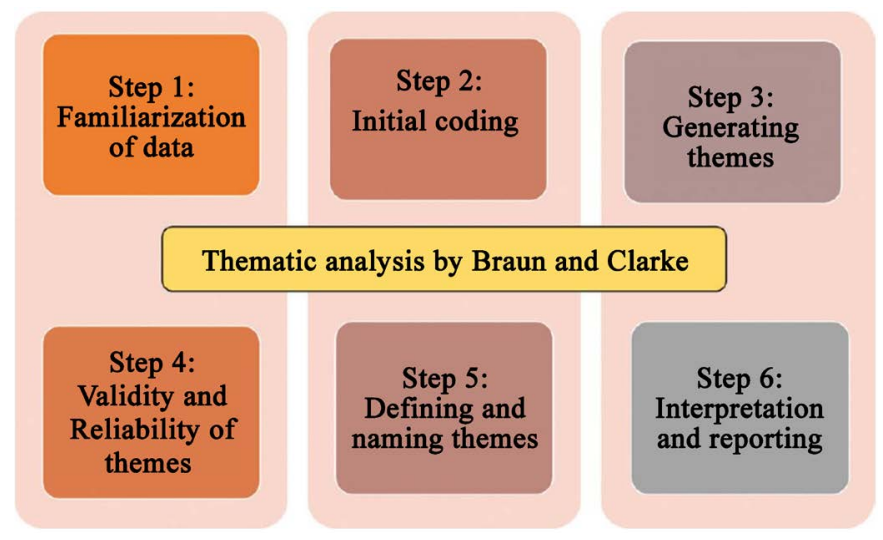

Figure 1. Thematic Analysis by Braun and Clarke. Source: Braun and Clarke (2006) [10]. 
occupation, religion, and interview category as presented in Tables 1-4. A total of 20 participants were interviewed; 15 were males and 5 were females. The ages of the participants ranged from 18 to 57 years with the average age being 31 years standard deviation of 10.92 . Seventy percent of the participants were between ages 18 - 33 years with age group 42 - 49 being the lowest percentage (10\%). The participants had occupations ranging from businessmen to health workers while some were students and unemployed. Nine (45\%) of the participants were businessmen and women while students and self-employed were three each i.e. (15\%) each, only one participant (5\%) was unemployed. Health

Table 1. Age and gender distribution of the participants.

\begin{tabular}{ccccc}
\hline & & Male & Female & Total \\
\hline Age & $18-25$ & 5 & 2 & 7 \\
$26-33$ & 6 & 1 & 7 \\
$34-41$ & 1 & 0 & 1 \\
& $42-49$ & 1 & 1 & 2 \\
& $50-57$ & 2 & 1 & 3 \\
& & 15 & 5 & 20 \\
\hline
\end{tabular}

Table 2. Occupation and gender of the participants.

\begin{tabular}{ccccc}
\hline & & Male & Female & Total \\
\hline Occupation & Student & 2 & 1 & 3 \\
& Businessman & 8 & 1 & 9 \\
& Self-Employed & 3 & 0 & 3 \\
& Unemployed & 0 & 1 & 4 \\
& Health Worker & 2 & 2 & 20 \\
\hline
\end{tabular}

Table 3. Religion and sex of the participants.

\begin{tabular}{lcccc}
\hline & & & \multicolumn{2}{c}{ Sex } \\
\cline { 3 - 5 } & & & Female & Total \\
\hline \multirow{2}{*}{ Religion } & Islam & 15 & 3 & 18 \\
& Christianity & 0 & 2 & 2 \\
\multicolumn{2}{c}{ Total } & 15 & 5 & 20 \\
\hline
\end{tabular}

Table 4. Participants' interview category.

\begin{tabular}{cc}
\hline & $\mathrm{N}$ \\
\hline Focus Group Discussion & 16 \\
Key Informant Interview & 4 \\
Total & 20 \\
\hline
\end{tabular}


workers accounted for about $20 \%$ (4) of the participants.

There were two interview participant categories: 16 (80\%) of the participants were interviewed using FGDs, while 4 (20\%) participants were Key Informants.

Responses were grouped into three sub-themes: factors contributing to treatment adherence, patient behavioural patterns that engender treatment adherence and effects of treatment adherence on treatment outcomes.

\subsection{Barriers and Facilitators of Adherence to Treatment}

The study participants mentioned factors they thought contributed to treatment adherence. Some of the listed factors included effective and appropriate communication by healthcare workers with patients, availability of competent treatment supporters, social support from relatives and communities, side effects from drug usage and availability of effective transportation to the facilities. In the exact words of one of the key informants, a female healthcare worker of one of the treatment facilities:

"Manner of approach by health personnel goes a long way in ensuring treatment adherence, putting a call to patients, making them feel loved and cared for, it goes a long way in motivating the patients to use their drugs" (Female Health worker, KI).

Another respondent, a cured patient, also mentioned that having a good treatment supporter who lives close-by and is very supportive contributes to better treatment adherence. In his own words:

"The support I get from my mother has been a key factor in helping me adhere to my drugs, she ensures I use my drug as at when due and cares for me making my wellbeing her concern" (34-year-old Businessman, FGD).

A 22-year old female student who has completed treatment mentioned:

"I am grateful to my mother as my treatment supporter as she stood by me through out, she kept reminding me to use my drug and provided other moral and financial support, this has been the key part of my treatment adherence" (22-year-old Female Student, FGD).

Social support from relatives and communities also contributed to treatment adherence. One of the respondents mentioned in her words that:

"Understanding and supportive husband and community members help in treatment adherence while difficult and unsupportive husband or relatives do not" (Female Health worker, KI).

Side effects from drug usage could also be a limiting factor for treatment adherence. A respondent said:

"At a point, because of the side effects of the drug, I almost stopped using my drugs, but after counselling from the health worker and continued reduction in the side effects I continued taking my medication and now I am 
cured" (34-year-old Business man, FGD).

Respondents mentioned that distance from health facilities affected treatment adherence and farther distances created transportation cost burden. A respondent who was on treatment mentioned his challenge regarding transportation to health facilities which he stated in his words as follows:

"I have experienced difficulty transporting myself to the health facility sometimes no money but most times I have to save enough to cater for my transport to ensure that I don't run out of drugs" (34-year-old Business man, FGD).

\subsection{Behavioural Patterns that Engender Treatment Adherence}

The participants were asked to discuss the behavioural patterns that engender treatment adherence and their responses included: A positive mind set towards drug usage and treatment, support and motivation from health worker, feeling of wellness during treatment and regular consumption of balanced diets.

One of the respondents, a female health worker in one of the treatment facilities stated that a positive mind-set is key to treatment adherence:

"For me I believe having a positive mind-set towards drug usage and treatment generally contributes to treatment adherence, most times patients with positive mind-sets tend to adhere better than those without" (Female Health Worker, KI).

Another respondent who was on treatment mentioned that support and care that came from health workers is a motivating factor for treatment adherence:

"I feel motivated to use my drugs when the health worker shows care and support and responds to my questions promptly" (27-year-old Male Self-employed, FGD).

One of the respondents also mentioned that the feeling of wellness during treatment motivates him to adhere to treatment. He opined that the feeling of wellness motivates him to complete his treatment especially when equipped with the knowledge of the duration of treatment. In his words he said:

"Since I know the duration of my treatment, whenever I see changes and feelings of wellness it motivates me to complete using my drug as I am assured of being cured" (30-year-old Male self-employed, FGD).

Eating good food and a balanced diet was cited as a key part of treatment which could quicken recovery by three respondents who were already cured. In his words, one of them said:

"Every time I eat good food and use my drugs, I feel better and get a feeling that my healing would be fast and it motivates me to use my drugs daily" (22-year-old Female Student, FGD). 


\subsection{Effects of Treatment Adherence on Treatment Outcome}

When asked about the effects of treatment adherence on treatment outcome, all the respondents said that it positively leads to a better treatment outcome. A female health worker (a DOTS Officer) in one of the treatment facilities said:

"It is evident that treatment adherence contributes perfectly to treatment outcome, there are so many examples of patients that adhered strictly to their drugs and are now cured in my health facility where I work" (Female Health Worker, KI).

Another respondent, a cured female patient, while also agreeing, said:

"For me I know treatment adherence contributes immensely to positive treatment outcome as I am a living testimony" (25-year-old Female Unemployed, FGD).

\subsection{Discussion}

This study provides information on the factors that contribute to tuberculosis treatment adherence as well as behavioural patterns that engender treatment adherence. The study found out that good and effective communication by healthcare personnel, having a competent treatment supporter, social support from relatives and communities, side effects from drugs, low cost of transportation to treatment facilities; positive mind set towards treatment, support and motivation from health worker, feeling of wellness during treatment and the consumption of a balanced diet, all contribute to treatment adherence.

It was observed that effective and sustained communication by health personnel could foster patients' adherence to treatment. TB patients who have a good relationship with health workers tend to adhere to their drug regimen better than those who do not. Health personnel who are friendly and accommodating would keep their patients in treatment better than those who are hostile. This observation is similar to the findings in a study done in Asmara, Eritrea [11].

The availability of an effective and supportive treatment supporter who is closely related and lives close to the patient is instrumental in improving TB treatment adherence [12]. This study found that the reason for most non-adherence by patients is due to the poor treatment support. Therefore, having an effective and caring treatment supporter could be a game changer. Similar studies conducted in $\mathrm{Na}$ mibia and South Africa by Tuhadeleni et al., (2016) [13] and Duwell et al., (2013) [14] respectively, found out that roles of treatment supporters are pivotal to ART treatment adherence. They found out that, in addition to assistance with medications, treatment supporters have the capacity to promote healthy behaviours and provide other types of support, which may contribute to improved outcomes, particularly with enhanced training.

Good social support from relatives and community members devoid of stigma and discrimination could foster treatment adherence and help keep patients on 
retention. The involvement of relatives and community members who are supportive and interested in patients' well-being in TB treatment could greatly improve patients' TB treatment adherence as affirmed by our respondents. In a study among adult and paediatric TB patients in Peru, the participants stated that social and psychosocial support from social groups including family, friends, and community members, motivated them to adhere to their treatment regimen [15].

Also, side effects from drug usage could affect patients' treatment adherence. Patients experiencing severe drug side effects sometimes attempt to interrupt or stop treatment entirely due to these effects while those patients experiencing mild side effects with improved health conditions tend to stick with their drugs. Patient education could improve response to drug side effects by equipping patients with information of what to expect and how to deal with the side effects early on. Two studies conducted in Southern Ethiopia also had similar findings [16] and [17].

TB patients who live close to health facilities and who do not incur transportation costs tend to adhere to their drugs since there would not be treatment interruption due to financial incapacity. Participants mentioned that staying close to their health facilities have contributed to their treatment adherence. Some patients who stay far from their treatment site also stated that they had to save more to cater for their transportation costs. This implies that situating TB treatment sites close to the people through a primary healthcare (PHC) Model or mobile treatment site model could improve treatment adherence. These findings are like those documented in studies from Indonesia [18] and [19].

This study identified some behavioural patterns that engender treatment adherence hence helped patients stick to their TB drugs. A positive mind-set towards treatment was indicated as a crucial behavioural pattern that can influence treatment adherence. Patients with a positive mind-set and optimism to get cured would adhere better to treatment than those without. Also, support and motivation from health workers can influence patients' adherence while helping them stay in retention.

Contrary to the findings in a study conducted in Zambia by Kaona et al., (2004) [20] where it was observed that TB patients failed to comply to the TB drug regimen once they started feeling better, this study found out that the feeling of wellness during treatment could serve as a motivating factor for treatment adherence provided that patients knew their treatment duration. When patients feel well during treatment and are equipped with the right information on treatment duration, they tend to stay motivated and focus on getting completely cured.

Consumption of good food and balanced diets has been cited as a key factor that could improve treatment outcomes and support treatment adherence [15]. However, most patients with little or no income could find it challenging to get good food thereby increasing their risk of treatment interruption and 
non-adherence. Studies conducted in Uganda and the United States by Weiser et al., (2010) [21] and Young et al., (2014) [22] respectively, established a linkage between food insecurity and non-adherence of anti-retroviral drugs in HIV \& AIDS treatment. They found out that food insecurity is a common and significant barrier to accessing medical care and ARV adherence. Therefore, strategies should be in place to support rural and poor patients and ensure access to good food as this could improve their retention and treatment outcome. In Peru, the National TB program established a food aid for patients, especially those with low socioeconomic status [15].

This study has assessed factors influencing adherence to tuberculosis treatment in selected health facilities in Kano State. However, this study is not generalizable due to its qualitative nature, but the findings could provide in-depth understanding of the current situation and help policy and programme decision making. Further, nationwide, preferably quantitative, studies should be conducted to broaden the current understanding of the factors associated with TB treatment adherence in Nigeria.

\section{Conclusion}

This study showed that factors such as; good and effective communication by health personnel, availability of competent treatment supporters, social support from relatives and communities, minimal side effects from TB drug use and low transportation cost to health facilities contributed to treatment adherence., It was also found that behavioural patterns such as; a positive mind set towards treatment, support and motivation from health workers, feeling of wellness during treatment, complete and balanced diet all engender treatment adherence which impacts positively on treatment outcomes. For better treatment adherence, an experienced and effective treatment supporter should be selected by TB patients, health workers should ensure effective communication and maintain friendly relationships with TB patients. TB patients should be properly counselled before being commenced on treatment to help them maintain a positive mind-set towards treatment while also supporting them socially and financially especially in the provision of good food and treatment support.

\section{Conflicts of Interest}

The authors declare no conflicts of interest regarding the publication of this paper.

\section{References}

[1] WHO (2020) Tuberculosis Country Profile: Nigeria. https://worldhealthorg.shinyapps.io/tb_profiles/?_inputs_\&lan=\%22EN\%22\&iso2= $\% 22 \mathrm{NG} \% 22$

[2] WHO (2019) Global Tuberculosis Report. World Health Organization, Geneva.

[3] National Population Commission (2010) 2006 Population and Housing Census. Priority Table. 
[4] National Tuberculosis and Leprosy Control Programme (NTBLCP) (2018) NTBLCP Annual Report.

[5] United Nations General Assembly (2017) Options and Modalities for the Organization of the High-Level Meeting on Tuberculosis Convened by the President of the General Assembly.

[6] Ibrahim, L.M., Hadejia, I.S., Nguku, P., Dankoli, R., Waziri, N.E., Akhimien, M.O., Ogiri, S., Oyemakinde, A., Dalhatu, I., Nwanyanwu, O. and Nsubuga, P. (2011) Factors Associated with Interruption of Treatment among Pulmonary Tuberculosis Patients in Plateau State, Nigeria. Pan African Medical Journal, 17, 78. https://doi.org/10.11604/pamj.2014.17.78.3464

[7] Adejumo, O.A., Daniel, O.J., Otesanya, F.A., Ashipa, T., Adejumo, E.N. and Abdur-Razzaq, H.A. (2016) Factors Affecting Treatment Interruption among TB Patients in Lagos Nigeria: Is There Any Need for Treatment Supporters? International Journal of Clinical Medicine, 7, 389-398. https://doi.org/10.4236/ijcm.2016.76042

[8] National Population Commission and National Bureau of Statistics Estimates (2016) National Population Estimates.

https://nigeria.opendataforafrica.org/xspplpb/nigeria-census

[9] SHOPS Plus Project (2016) Ending Tuberculosis in Nigeria through Provider Networks and Behavior Change. Newsletter.

https://www.shopsplusproject.org/article/ending-tuberculosis-nigeria-through-prov ider-networks-and-behavior-change

[10] Braun, V. and Clarke, V. (2006) Using Thematic Analysis in Psychology. Qualitative Research in Psychology, 3, 77-101. https://doi.org/10.1191/1478088706qp063oa

[11] Gebreweld, F.H., Kifle, M.M., Gebremicheal, F.E., Simel, L.L., Gezae, M.M., Ghebreyesus, S.S., Mengsteab, Y.T. and Wahd, N.G. (2018) Factors Influencing Adherence to Tuberculosis Treatment in Asmara, Eritrea: A Qualitative Study. Journal of Health, Population and Nutrition, 37, 1. https://doi.org/10.1186/s41043-017-0132-y

[12] WHO (2010) Treatment of Tuberculosis Guidelines. http://ncbi.nlm.nih.gov/books/NBK138748/pdf/Bookshelf_NBK138748.pdf

[13] Tuhadeleni, O., Opotamutale, D. and Nuuyoma, V. (2016) The Perceptions of HIV-Positive Patients (ART Patients) on Anti-Retroviral Therapy (ART), Treatment Supporters and Health Care Workers with Regard to Their Role in ART Adherence at ART Clinics in the Intermediate Hospital Oshakati, Namibia. Health Science Journal, 10, 14. https://doi.org/10.14419/ijm.v5i1.6999

[14] Duwell, M.M., Knowlton, A.R., Nachega, J.B., Efron, A., Goliath, R., Morroni, C., Maartens, G. and Chaisson, R.E. (2013) Patient-Nominated, Community-Based HIV Treatment Supporters: Patient Perspectives, Feasibility, Challenges, and Factors for Success in HIV-Infected South African Adults. Aids Patient Care and STDs, 27, 96-102. https://doi.org/10.1089/apc.2012.0348

[15] Paz-Soldán, V.A., Alban, R.E., Jones, C.D., et al. (2013) The Provision of and Need for Social Support among Adult and Pediatric Patients with Tuberculosis in Lima, Peru: A Qualitative Study. BMC Health Services Research, 13, 290. https://doi.org/10.1186/1472-6963-13-290

[16] Boru, C.G., Shimels, T. and Bilal, A.I. (2017) Factors Contributing to Non-Adherence with Treatment among TB Patients in Sodo Woreda, Gurage Zone, Southern Ethiopia: A Qualitative Study. Journal of Infection and Public Health, 10, 527-533. https://doi.org/10.1016/j.jiph.2016.11.018

[17] Gube, A.A., Debalkie, M., Seid, K., Bisete, K., Mengesha, A., Zeynu, A., Shimelis, F. and Gebremeskel, F. (2018) Assessment of Anti-TB Drug Nonadherence and Asso- 
ciated Factors among TB Patients Attending TB Clinics in Arba Minch Governmental Health Institutions, Southern Ethiopia. Tuberculosis Research and Treatment, 2018, Article ID: 3705812. https://doi.org/10.1155/2018/3705812

[18] HerreroI, M.B., Ramos, S. and ArrossiI, S. (2015) Determinants of Non-Adherence to Tuberculosis Treatment in Argentina: Barriers Related to Access to Treatment. Revista Brasileira de Epidemiologia, 18, 287-298. https://doi.org/10.1590/1980-5497201500020001

[19] Ruru, Y., Matasik, M., Oktavian, A., Senyorita, R., Mirino, Y., Tarigan, L.H., Werf, M.J., Tiemersma, E. and Alisjahbana, B. (2018) Factors Associated with Non-Adherence during Tuberculosis Treatment among Patients Treated with DOTS Strategy in Jayapura, Papua Province, Indonesia. Global Health Action, 11, Article ID: 1510592. https://doi.org/10.1080/16549716.2018.1510592

[20] Kaona, F., Tuba, M., Siziya, S. and Sikaona, L. (2004) An Assessment of Factors Contributing to Treatment Adherence and Knowledge of TB Transmission among Patients on TB Treatment. BMC Public Health, 4, 68.

https://doi.org/10.1186/1471-2458-4-68

[21] Weiser, S.D., Tuller, D.M., Frongillo, D.A., Sekungu, J., Mukiibi, N. and Bangsberg, D.R. (2010) Food Insecurity as a Barrier to Sustained Antiretroviral Therapy Adherence in Uganda. PLoS ONE, 5, e10340.

https://doi.org/10.1371/journal.pone.0010340

[22] Young, S., Wheeler, A.C., McCoy, S.I. and Weiser, S.D. (2014) A Review of the Role of Food Insecurity in Adherence to Care and Treatment among Adult and Paediatric Populations Living with HIV and AIDS. AIDS and Behavior, 18, S505-S515. https://doi.org/10.1007/s10461-013-0547-4 


\section{Appendix 1. Focus Group Discussion Interview Guide}

\section{Question Guide}

1) What do you know about TB treatment?

2) How long have you been on TB treatment?

3) At what time do you miss using your drug or treatment appointment?

4) What attributed to your missing your drug or appointment?

5) If you did not miss, what influenced your adherence to your drugs and appointment?

6) What factors contributed to your treatment adherence?

7) What pattern of behaviour helps you keep to your drug appointment? Tell us more about it.

8) Has treatment adherence contributed to your treatment outcome?

9) What can you say about the effect of adhering to your drug on your health (treatment outcome)?

10) Would you advice others to follow your behaviour pattern for treatment adherence?

\section{Appendix 2. Key Informant Interview Guide}

\section{Question Guide}

1) Can you tell us about your role on the TB treatment in your facility?

2) What factors would you say contributed to your patents treatment adherence?

3) What behaviour pattern do you think supports treatment adherence?

4) In your opinion do you think treatment adherence contribute to positive treatment outcome?

5) What advice will you give to current patients on treatment? 